\begin{tabular}{|c|c|}
\hline & $\begin{array}{l}\text { International Journal of Trend in Scientific } \\
\text { Research and Development (IJTSRD) }\end{array}$ \\
\hline 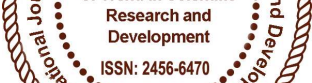 & International Open Access Journal \\
\hline 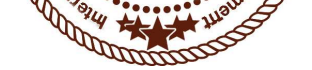 & ISSN No: 2456 - 6470 | www.ijtsrd.com | Volume - 2 | Issue - 2 \\
\hline
\end{tabular}

\title{
Effect of Necktie on Heart Rate, Blood Pressure and Ankle Brachial Index among Male Office Workers
}

\author{
Dr. Sohajpreet Kaur (PT), Dr. Reecha Bedi (PT), Dr. Suresh Mani (PT) \\ Department of Physiotherapy, \\ Lovely Professional University, Phagwara, Punjab, India
}

\section{ABSTRACT}

Aim: To evaluate the effect of a necktie on heart rate (HR), blood pressure (BP) and ankle brachial index (ABI)

Methods: In a cross-sectional study, a total of 56 clients were enrolled of age group 25-35 years (groupA) and 36-45 years (group-B) wearing necktie for more than 1 year. Heart rate (carotid pulse), blood pressure and ABI of the clients were measured by Oscillometric Sphygmomanometer.

Results: There was a significant difference in $\mathrm{HR}$ of group $\mathrm{A}$ as compared to group $\mathrm{B}$, no significant difference was seen in systolic blood pressure (SBP) of group-A but some significant difference was demonstrated in group-B. For diastolic blood pressure (DBP), there was much significant difference seen in group-B as compared to group-A. There was no significant change seen for $\mathrm{ABI}$ in any of the groups.

Conclusion: A necktie may increase heart rate and decreases blood pressure in both the age groups but have no effect on ABI.

Keywords: Necktie, heart rate, carotid pulse, systolic blood pressure, diastolic blood pressure, ankle brachial index.

\section{Introduction}

Traditionally, the necktie is being the significant mark of office attire, especially for professionals, it is worn over the neck where major neurovascular structures are present. Carotid sinus is a slight dilatation where the common carotid artery bifurcates into internal and external carotid artery in the mid neck region. The walls of the carotid sinus contain the receptors sensitive to change in blood pressure. Baroreceptors are the sensory neuro-mechanoreceptors which excites when blood vessel gets stretched, so that if arterial pressure suddenly increases, the walls of these vessels will dilate, which increases the firing frequency of action potential generated by baroreceptors. Although the baroreceptors respond to rise or decline in systemic arterial pressure, their most important work responds to a sudden decrease in arterial pressure. According to Mark Rafferty et al (2011), a necktie may increase intracranial pressure by creating a direct pressure over jugular veins which effects venous return [1]. Similarly, another study reported the possibilities of increase in intraocular pressure due to necktie [2]. However, there is no study which states the effect of a necktie on cardiovascular parameters.

Necktie puts pressure over the carotid sinus, so it may affect various cardiovascular parameters like heart rate, blood pressure, and ABI. Physiologically, compression on carotid sinus leads to falling in carotid sinus pressure which increases heart rate and fall in blood pressure [3]. As the systolic pressure of arm decreases, the ABI will decrease and vice-versa. In normal subjects, the pressure at the ankle is slightly higher than at the elbow. Since few studies reported the effect of wearing a necktie on various body systems like it increases the intraocular pressure [2] and negative effects on cerebrovascular functions [1]. However, there were no earlier studies which address effects of a necktie on cardiovascular parameters. 
Therefore, the aim of this study was to find the effect of wearing a necktie on heart rate, blood pressure and ABI among male office workers.

\section{Methodology}

\section{Design \& Setting}

The study was a multi-centre, two-group, crosssectional study among office workers aged between 25-45 years, wearing a necktie for more than one year. Fifty-six clients were recruited over the time period of six months and each client was assessed for one day in study, in which they all were asked to fill the questionnaire and were assessed further according to the parameters. This project was approved by Project Approval Committee of Lovely Professional University, Phagwara. Clients were recruited from multi-specialty hospitals located in Raikot and Ludhiana (Punjab).

\section{Participants}

Clients were recruited as per the inclusion criteria. They were office workers/professionals, who were able to read and write in English. The clients who were fully willing to participate were considered and the written consent was filled by them with their contact number and address. A semi-structured questionnaire was filled by the clients which represent their health and the present, past and future lifestyle. The exclusion criterions of this study were: those who were pre-diagnosed with hypo or hypertension, those who had any cardiovascular disease. Clients who were having any peripheral vascular disease were not eligible to participate in this study.

\section{Instruments}

A semi-structured questionnaire was used to get information regarding the lifestyle, experiences, medical and surgical profile of the clients. An Oscillometer Sphygmomanometer [4] (Diamond) and the stethoscope were used for measuring blood pressure on brachium and ankle. A stopwatch (android mobile application) was used to calculate HR (carotid pulse) from the carotid artery.

\section{Procedure}

Within a hospital set-up, clients were approached and selected as per the inclusion criteria. Aim and procedure of the cardiovascular assessment were told to the clients and were asked to sign the consent form after reading it. Clients were asked about the demographic data which included name, age, gender, occupation, place and contact number. A semistructured questionnaire was given to clients related to the medical and surgical background, current health problem and duration of wearing necktie along with query about the problems faced by clients because of the necktie. After that, clients were asked to be in sitting position for cardiovascular assessment. A cuff was tied on the brachium and inflated, the blood pressure was measured using an oscillometric sphygmomanometer [4]. Similarly, HR was measured using carotid pulse rate by placing two fingers on carotid artery between the larynx and anterior border of sternocleiodomastoid muscle while client in sitting position with chin elevated. The pressure was applied until the maximum pulsation felt and the number of pulses felt in a minute was calculated using a stopwatch [5]. Lastly, ABI of the client was calculated by measuring blood pressure at the ankle and dividing the systolic value of blood pressure of the ankle to the SBP of the brachium [4].

\section{Statistical Analysis}

The statistical analysis was done using SPSS software version 20.0 (SPSS Inc., Chicago, IL, 2011). The data was normally distributed based on testing normality. The mean and standard deviation of HR, SBP and DBP and ABI were calculated. One 'sample-t' test was used to compare the mean difference between both the groups of each parameter with a significant level of $0.05(\mathrm{p} \leq 0.05)$.

\section{Results}

Fifty-six clients were enrolled and divided into two age groups, group-A (66\%) aged between 25 and 35 years, while group-B (34\%) aged between 36 and 45 years. The mean HR of group-A $(80.03 \pm 10.22)$ and Group-B (76.74 \pm 6.91$)$ were higher than normal reference HR value of 72.5 for group-A and 73 for group-B. Based on One 'sample-t' test, a statistically significant mean difference of $7.53(95 \% \mathrm{CI}=4.1$ to $\left.10.9, \mathrm{t}_{(36)}=4.48, p=0.00\right)$ for group-A and $3.74(95 \%$ $\mathrm{CI}=0.4-7.0, \mathrm{t}_{(18)}=2.35, p=0.03$ ) for group-B.

The mean SBP of Group-A (122.46 \pm 18.71$)$ was higher than normal reference score of $121.5 \mathrm{mmHg}$, whereas, mean value of group-B (108.05 \pm 9.92) was lower than normal reference score of $124 \mathrm{mmHg}$. There was a statistically significant mean difference of $0.26(95 \% \mathrm{CI}=-20.7$ to -11.16$), \mathrm{t}_{(18)}=-7.004, p=$ 
$0.00)$ for group-B than mean difference $0.96(95 \% \mathrm{CI}$ $=-5.28$ to $\left.7.20, \mathrm{t}_{(35)}=0.313, p=0.76\right)$ for the group-A. The mean DBP of group-A $(77.03 \pm 12.44)$ and Group-B (75.11 \pm 12.46$)$ was lower than DBP score of 81.5 for group A and 82.5 for group-B. There was a statistically significant mean difference of $-4.47(95 \%$ $\mathrm{CI}=-8.6$ to $0.32, \mathrm{t}_{(36)}=-2.19, p=0.035$ for Group $\mathrm{A}$ and $7.39(95 \% \mathrm{CI}=-13.40$ to -1.4$), \mathrm{t}_{(18)}=-2.68, p=$ $0.02)$. The mean ABI of both groups $(1.1 \pm 0.97)$ was equal to the normal ABI score of 1.1 (Table 1).

\section{Discussion}

This study was aimed to evaluate the effects of wearing a necktie on cardiovascular parameters as it significantly compresses the neck area (carotid sinus). Direct pressure over the carotid sinus could lead to compression of the internal carotid artery and jugular vein which excites the baroreceptors. Baroreceptors are the mechanoreceptors which excites when blood vessel gets stretched, so that if arterial pressure suddenly increases, the walls of these vessels will dilate, which increases the firing frequency of action potential generated by baroreceptors. Baroreceptors respond to rise or decline in systemic arterial pressure, but mainly respond to a sudden decrease in arterial pressure. The baroreflex responds to acute changes in blood pressure of the carotid artery. If the blood pressure of the person decreases, it is sensed by baroreceptors as a decrease in tension, and then it will lower down the firing of impulses. This causes the vasomotor center to assist the sympathetic activity in the heart and blood vessels, causing an increase in HR.

A previous study reported that the compression or massage of carotid sinus may induce a drop in the blood pressure and increases in HR [3]. Sufficient evidence is reported in the literature to support that a necktie could affect the intraocular pressure [2], [6], and cervical spine range of motion and muscle activity [7] and causes syncope [8]. However, there was no study which reports that how a necktie could alter the cardiovascular parameters. We hypothesized that the blood pressure decreases and the HR increase due to compression of carotid sinus in the neck due to the necktie. The results of the current study support that there was the highly significant difference in HR of group A and Group B from the normal reference values. Similarly, there was a significant difference was seen in DBP in both Group A and group B, whereas a significant difference in SBP was demonstrated in group B, but not in the group A. In addition, there was no significant difference seen for $\mathrm{ABI}$ in any of the cases. Therefore, the study results support the necktie might affect cardiovascular parameters among male office workers.

The small sample size and considering only male participants was the limitation. Secondly, there was no comparative group of normal individuals and no inclusion of demographic data like height and weight. Therefore, the recommendation for future study is to take large sample size including females. A control group of normal individuals could be taken, including their height and weight as demographic parameters along with the cardiovascular parameters.

\section{Conclusion}

This study concluded that a necktie might alter the HR and DBP for both age young and middle age groups and affect SBP for older age group. However, a necktie does not affect the SBP for younger group. Similarly, there is no effect of a necktie on ABI as well among both young and older groups. Therefore, it is recommended that people who are wearing a necktie on regular basis should lose the tie in between to release the continuous pressure on the neck to prevent unwanted adverse cardiovascular events.

\section{Acknowledgement}

The authors like to extend sincere thanks to all the clients who participated in this study and Dr. Durga Prathap for helping us throughout the project and making this project successful. Also, we would like to thank Mr. Gurwinder Singh for assisting in client recruitment and his support to complete the project.

\section{Disclosure statement}

There is no potential conflict of interest to disclose.

\section{Contribution of each author}

Sohajpreet Kaur: Involved in client recruitments, data collection, data analysis and wrote manuscript.

Reecha Bedi: Involved in conception of research idea, design and supervision of entire study.

Suresh Mani: Data analysis, monitored results and proof reading and finalized the manuscript. 


\section{REFERENCE}

1. M. Rafferty, T. J. Quinn, J. Dawson, and M. Walters, "Neckties and Cerebrovascular Reactivity in Young Healthy Males: A Pilot Randomised Crossover Trial," vol. 2011, 2011.

2. R. R. C Teng, R Gurses-Ozden, J M Liebmann, C Tello, "Effect of a tight necktie on intraocular pressure," Br. J. Ophthalmol., vol. 87, no. 2003, pp. 946-948, 2003.

3. E. J and D. Parry, "Some observations on the effects of stimu- lating the carotid arterial stretch receptors in the carotidartery of man (abstract).," J. Physiol., vol. 137, p. 45, 1957.

4. Á. Herráiz-Adillo, V. Martínez-Vizcaíno, I. Cavero-Redondo, C. Álvarez-Bueno, M. GarridoMiguel, and B. Notario-Pacheco, "Diagnostic accuracy study of an oscillometric ankle-brachial index in peripheral arterial disease: The influence of oscillometric errors and calcified legs," PLoS One, vol. 11, no. 11, pp. 1-16, 2016.
5. D. C. Morris, "The Carotid Pulse," Clin. Methods Hist. Phys. Lab. Exam., 1990.

6. P. Talty and P. D. O'Brien, "Does extended wear of a tight necktie cause raised intraocular pressure?," J. Glaucoma, vol. 14, no. 6, pp. 50810, Dec. 2005.

7. I. G. Yoo, M. H. Kim, and W. G. Yoo, "Effects of wearing a tight necktie on cervical range of motion and upper trapezius muscle activity during computer work," Work, vol. 39, no. 3, pp. 261266, 2011.

8. N. C. Munro, S. McIntosh, J. Lawson, C. A. Morley, R. Sutton, and R. A. Kenny, "Incidence of complications after carotid sinus massage in older patients with syncope.," J. Am. Geriatr. Soc., vol. 42, no. 12, pp. 1248-51, Dec. 1994.

\begin{tabular}{|l|l|l|l|l|}
\hline \multirow{2}{*}{ Heart Rate } & \multicolumn{2}{|c|}{$<35$ Years $(\mathrm{n}=37)$} & \multicolumn{2}{c|}{$>35$ Years $(\mathrm{n}=19)$} \\
\cline { 2 - 5 } & Mean \pm SD & "t" value & Mean \pm SD & "t" value \\
\hline Systolic Pressure & $77.03 \pm 12.44$ & $4.47^{*}$ & $76.74 \pm 6.91$ & $2.35^{+}$ \\
\hline Diastolic Pressure & $122.46 \pm 18.71$ & 0.31 & $108.05 \pm 9.92$ & $-7.00^{*}$ \\
\hline Ankle-brachial Index $(\mathrm{n}=56)$ & $1.09 \pm 0.97$ & -0.05 & $1.09 \pm 0.97$ & -0.05 \\
\hline${ }^{*}=\mathrm{p}<0.01,{ }^{+}=\mathrm{p}<0.05, \mathrm{SD}=$ standard deviation & $77.03 \pm 12.44456$ & $-2.18^{+}$ & $75.11 \pm 12.46$ & $-2.58^{*}$ \\
\hline
\end{tabular}

Table 1 Mean and standard deviation of HR, systolic and diastolic blood pressure and ankle-brachial index among participants wearing a necktie. 